\title{
A Flying Robot with Adaptive Morphology for Multi-Modal Locomotion
}

\author{
Ludovic Daler, Julien Lecoeur, Patrizia Bernadette Hählen and Dario Floreano
}

\begin{abstract}
Most existing robots are designed to exploit only one single locomotion mode, such as rolling, walking, flying, swimming, or jumping, which limits their flexibility and adaptability to different environments where specific and different locomotion capabilities could be more effective. Here we introduce the concept and the design of a flying robot with Adaptive Morphology for Multi-Modal Locomotion. We present a prototype that can use its wings to walk on the ground and fly forward. The wings are used as whegs to move on rough terrains. This solution allows to minimize the structural mass of the robot by reusing the same structure (here the wings) for different modes of locomotion. Furthermore, the morphology of the robot is analysed and optimized for ground speed.
\end{abstract}

\section{INTRODUCTION}

There is a growing interest in the use of robots for applications such as exploration, search-and-rescue, or monitoring of the environment [1]. These robots have to deal with very complex terrains, such as semi-collapsed buildings, deep caverns, or forests with a lot of vegetation. Autonomous robots are appealing for these tasks due to their ability to explore areas that are risky and inaccessible to humans [2].

However, existing platforms have limited locomotion abilities. Most existing mobile robots exploit only one single locomotion strategy, such as rolling, walking, flying, hovering, climbing, swimming, crawling, or jumping. Operating in complex terrains is very challenging for mobile robots since the topology of the terrain could be subject to significant variations. Therefore, using only one locomotion strategy limits their flexibility and adaptability to different environments. For example, obstacles larger than the size of a robot represent a significant challenge for ground locomotion, but flying robots can easily overcome these obstacles. In a search-and-rescue scenario there is a need to fly quickly over extended areas, thereby the use of a winged robot is interesting. However, careful exploration in confined environments or near the ground is challenging for flying robots.

Terrestrial locomotion is more energy efficient over short distances; flying forward requires gaining speed, and hovering flight with a prop-hanging system consumes a lot of energy [3] and flapping even more [4]. For instance one can notice that birds and insects use their legs to move around over short distances and rest on the ground, but prefer to fly over long distances. For these reasons, a robot capable of ground locomotion and of flying-hovering locomotion is well suited to navigate in complex environments.

L. Daler, J. Lecoeur and D. Floreano are with the Laboratory of Intelligent Systems (http://lis.epfl.ch) at Ecole Polytechnique Fédérale de Lausanne (EPFL), CH1015 Lausanne, Switzerland (email: ludovic.daler@epfl.ch).

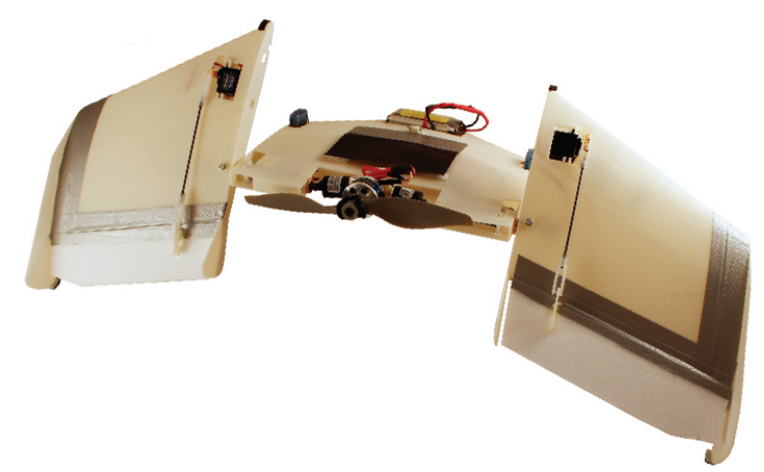

Fig. 1: $1^{\text {st }}$ prototype of the Deployable Air Land Exploration Robot (DALER) a flying and walking robot that uses its wings as whegs to move on the ground.

At first sight, multi-modal locomotion seems to be a good approach for improving the mobility of flying robots. However, the implementation of this solution into mobile robots showed that the addition of a second mode of locomotion on the platform could lead to a significant loss of performance of the two modes of locomotion if the two modes of locomotion do not share part of their structure or actuators [5], [6], [7], [8]. This effect is mainly caused by the weight added to the platform and the increased complexity of the robot. Therefore, it reduces the operating capabilities of each mode of locomotion and thus the overall mobility of the robot. For flying robots this disadvantage is even more significant, since increasing the weight could lead to the inability to take off.

Therefore, we propose a new approach, which we call "Adaptive Morphology 1 ", where parts of the structure of a robot are shared between the different modes of locomotion, instead of simply adding a second locomotion structure to an existing robot. Moreover, the structure could self-adjust the shape to adapt to the locomotion mode. The efficiency of locomotion in each mode of locomotion can be improved through adaptive morphology suitable for that mode. An example of an animal that uses adaptive morphology to improve its mobility is the salamander, which is capable of walking and rolling. Its body, originally shaped for walking, can take the shape of a large wheel to roll downhill [9]. Birds and other flying animals such as bats, use their wings for many different tasks, such as flying forward, stalling, hovering, squeezing through small openings, rolling over, walking, righting, and perching [10].

\footnotetext{
${ }^{1}$ In biology, morphology deals with the study of the form and structure of organisms and their specific structural features, such as aspects of the external appearance (shape, structure, pattern) and the form and structure of the internal parts.
} 
We aim to make adaptive deployable wings for improving the mobility of a flying robot, because having adaptive wings would provide many advantages. The wings are the largest components of the robot and their shape could be adaptively modified to augment efficiency of forward flight, hover flight, and displacement on the ground. For example, wings could be fully deployed for flying outdoors and reduced for hover flight and ground modes. Moreover, the wings structure could take the shape of whegs [11] in ground locomotion mode. Figure 2 shows a schematic representation of a flying robot capable of air and ground multi-modal locomotion (we called this robot DALER for Deployable Air Land Exploration Robot), and Fig. 1 shows the first prototype of this concept.

The design process that lead to this robot is presented in section III, along with two different prototypes that can use their wings to move on the ground using two different approaches. Then, the selected ground locomotion configuration is analysed and the morphology of the robot is modelled in a physics based simulator to optimize ground locomotion speed. Finally, the mechanical design of the DALER prototype that was built is presented in section IV. This prototype validates the concept of walking on the ground by using the wings as whegs. Finally, the flight capabilities of the two prototypes are analysed; in forward flight for the DALER and in hover flight for the other one.

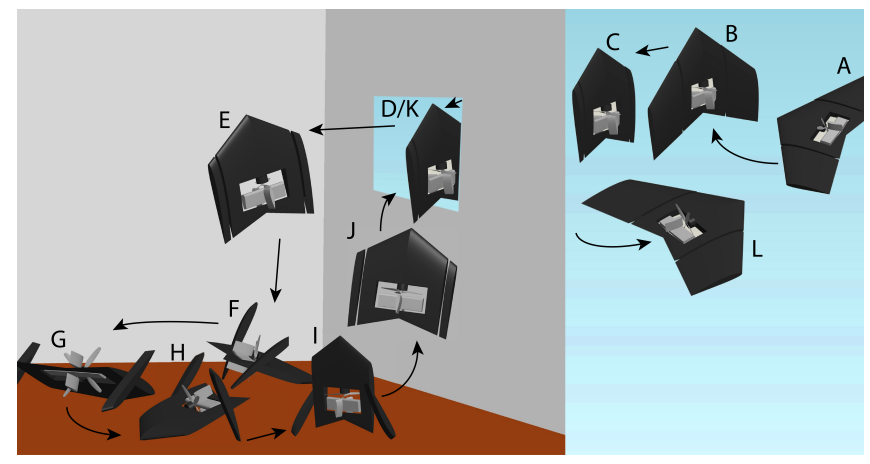

Fig. 2: Representation of the DALER flying robot that uses Adaptive Morphology for Multi-Modal Locomotion to perform a search-and-rescue mission. (A) Forward flight, (B) transition to hover flight, (C) reduce the wings' span, (D) enter a building trough a small opening, (E) hover indoors, (F) land on the ground, (G-H) move on the ground, (I) transition to hover flight, $(\mathrm{J})$ take-off, $(\mathrm{K})$ exit the building, and (L) transition back to forward flight.

\section{RELATED WORK}

Recently, some robots have been developed to display multiple modes of locomotion (e.g. hover-roll/walk, or flightroll/walk). Most of them are however optimized for one mode of locomotion and the second is often neglected. None of them reuse the same structure for different modes of locomotion in order to minimize weight and improve the effectiveness of each mode of locomotion.

BOLT [7] is a lightweight bipedal ornithopter capable of high-speed dynamic running and effecting transitions between aerial and terrestrial locomotion modes. This robot, due to its small size, can only run over small obstacles. A small ground robot designed by Kossett et al. [5], which has the ability to fly, utilizes a minimalistic wheeled ground mode to minimize weight, and a rotary-wing flight mode, enabling transformations at will. This design is ideal for hovering in an indoor environment and rolling on a flat ground, but it is not yet capable of rolling in rough terrains or flying over long distances. $M A L V$ [8] is a biologically inspired Micro Air-Land Vehicle. It flies using a chordwise, undercambered, bat-like compliant wing and walks over rough terrain using passively compliant whegs. MALV performs transition from flight to walking but most of the time cannot get back to the air, since it can only take off from the roof of a building of at least 6 meters high. The Japanese ministry of defense designed a spherical flying robot roughly the shape and size of a soccer ball. It has the capacity to land on the ground and roll, however, the ground locomotion is very limited since it can only roll passively on the ground to absorb the energy of the crash. MAVion Roll and Fly [12] is an aircraft with vertical take off and landing capability (VTOL), it has carbon fiber wheels to roll along grounds, walls and ceilings. Yet, the ground capabilities of this robot are limited since the wheels are only passive.

\section{DESIGN OF THE PLATFORM}

This section presents the selection and the design of the platform configurations for the ground and air locomotion modes. The selection of the type of configuration can be done from two different approaches; either we can start from a ground based platform and enable it with flying capabilities or start from a flying robot and make it move on the ground. Thus, we decided to analyze a search-and-rescue mission scenario in order to study the requirements of the robot. We expect that most of the distance travelled during the mission will be in the air. Consequently, we select first the type of airframe for the forward flight and look at how we could enable it with hovering and ground capabilities. Finally, the morphology of the robot is optimized with a simulator for the ground locomotion mode.

\section{A. Platform Requirements}

The first step of the design of a robot capable of multimodal ground and air locomotion is to define a list of mission specification such as flight distance, hover duration, ground distance, and payload capacity of the robot.

We based our analysis on a real life example such as the recent earthquake that happened in Haiti, in January 2010, which destroyed almost completely the city of Portau-Prince. When autonomous robots could have been used to localize people stuck in collapsed houses all around the city. In this scenario, the platform must be able to reach any point in the city starting from its center and come back. The radius of the city is approximatively $5 \mathrm{~km}$, giving a travel distance of $10 \mathrm{~km}$. The estimated time needed to enter and exit a house is around 5 minutes in total and the platform must have enough energy in order to move on the ground to explore 
a small house. Apart from fulfilling these requirements the robot must also be capable of vertical take off and landing and of passing through small openings (to enter/leave the house). It must be robust against landings on any terrain, able to transition from ground mode to hover flight, and able to transition from hover flight to forward flight. Furthermore, in order to be safe for the people around, the total mass must be minimized.

\section{B. Platform Type Selection}

Considering these mission requirements, systematic analysis of the different airframe configurations, hovering methods, and wing-aided ground locomotion solutions were performed. As mentioned before, the priority will be given to the forward flight mode of locomotion since we want to cover long distances quickly.

Regarding the type of airframe; flapping-wing have been excluded from the beginning because of their mechanical complexity. Lighter-than-air platforms have been left out as well because of their low payload capacity per volume [13]. Therefore, fixed-wing airframes remain as the most suitable choice for forward flight and rotary-wings for hover flight. The design of a flying robot usually begins with decisions on the kind and size of the airframe to be used, we used the methodology presented in Leven's thesis [14], where many possible configurations of fixed-wing airframes are presented. The "flying wing" configuration was selected for the forward flight configuration. This airframe's main characteristic is the absence of a fuselage and tail, making it simple to construct and robust to landings. The airframe is inherently stable and provides good manoeuvrability.

For the hovering mode of locomotion we can either use a multi-rotor configuration, a helicopter-like configuration, or a coaxial motor configuration for the generation of the vertical thrust. The multi-rotor and the helicopter-like configurations are ruled out because the span of the robot cannot be reduced in order to pass through small openings (see Fig. 2). From our experience [15], the configuration with a coaxial motor and control flaps gives a better manoeuvrability, a simpler control, and is lighter than a thrust vectoring configuration, and is thus selected. This solution was implemented in the prototype presented in Fig. 3.

For the ground locomotion mode, as motivated previously, we only use the existing structure in order to minimize the total weight. Therefore, the wings are used for the ground locomotion; they can either be closed around the robot as shown in Fig. 3 and used to roll on the side, or they can be used as whegs to walk forward, as in Fig. 4. The first solution has the advantage of protecting the center of the robot during hover but has limited ground locomotion capabilities since it can only go in one direction, and has limited steering abilities. The second solution is simple to control, can go at high speed, and is more manoeuvrable than the other configuration. Figure 4 shows a ground locomotion sequence of the DALER flying robot that uses its wings to walk on the ground.

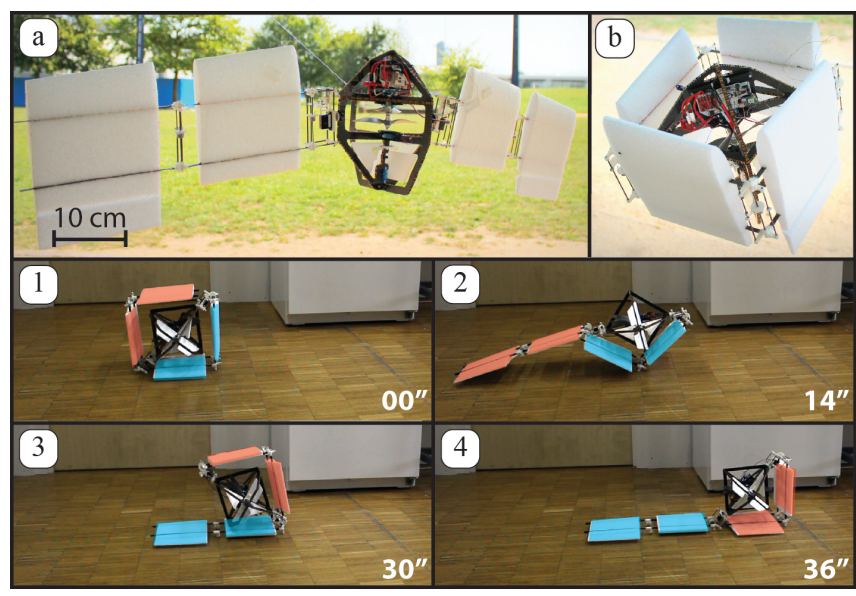

Fig. 3: Investigation of a prototype that can use its wings for rolling sideways. (a) Forward flight configuration. (b) Hover configuration. (1-4) Rolling sequence using the wings.

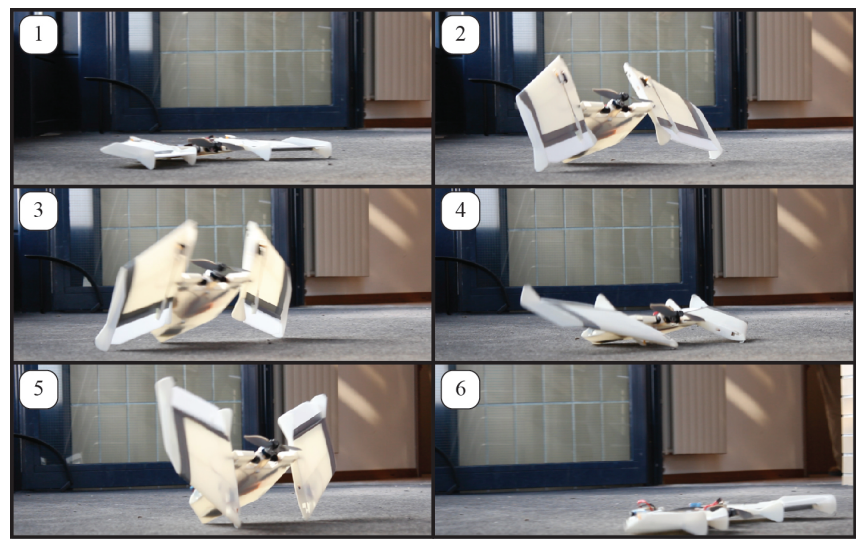

Fig. 4: Ground locomotion sequence of the DALER flying robot using its wings as whegs to walk on the ground, the entire sequence takes less than $4 \mathrm{~s}$.

\section{Ground Locomotion Analysis}

This subsection aims at analysing the ground locomotion of the DALER shown in Fig. 4. The geometry of a flying wing airframe can be described with 3 parameters (see Fig. 5); the "Taper ratio" $T=C_{t} / C_{r}$ (ratio between the tip chord $C_{t}$ and the root chord $C_{r}$ ), the "Swept angle" $\theta$, and the "half wing span" $b / 2$. For the ground locomotion we defined a new parameter that we called the "Inner ratio" $I=2 d / b$; the ratio between the distance $d$ (distance between the center of the robot and the rotating wing) and the half wing span. The position of the rotation axis of the wings $d_{\text {axis }}$ is defined from the front tip of the platform. In order to minimize the maximum torque in the motors that actuate the wings this length is constrained in order to have $w_{1}=w_{2}=w$. Finally, the position of the center of gravity (CG) is constrained, for flight stability reason, by the Mean Aerodynamic Chord (MAC), that depends on the lift distribution of the platform [16].

The first important point that we have to consider is that the center of the robot does not flip over when the robot 


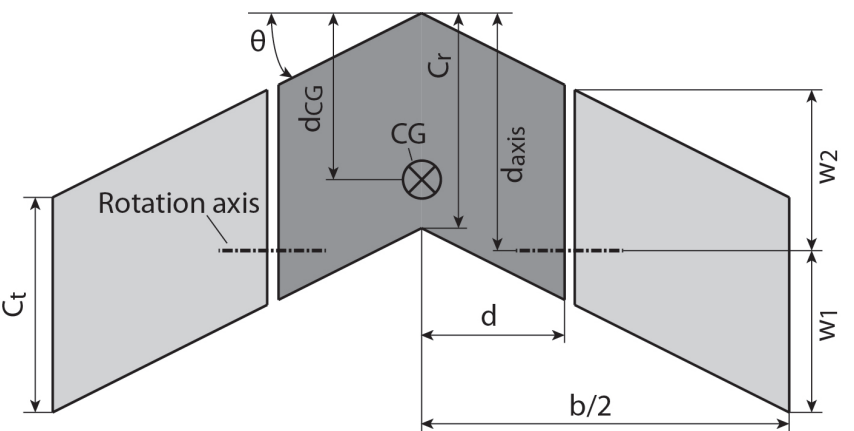

Fig. 5: Model of robot with dimension parameters.

is climbing a slope. Figure 6.a shows a schema of the robot seen from the side on a slope of angle $\beta$. To prevent flipping, the following condition has to be maintained:

$$
w<d_{\text {axis }} \cos (\beta) .
$$

Otherwise the center part of the robot would rotate instead of the wings, because the gravitational force would pull it backwards. Figure 6.b shows the corresponding limit situation.

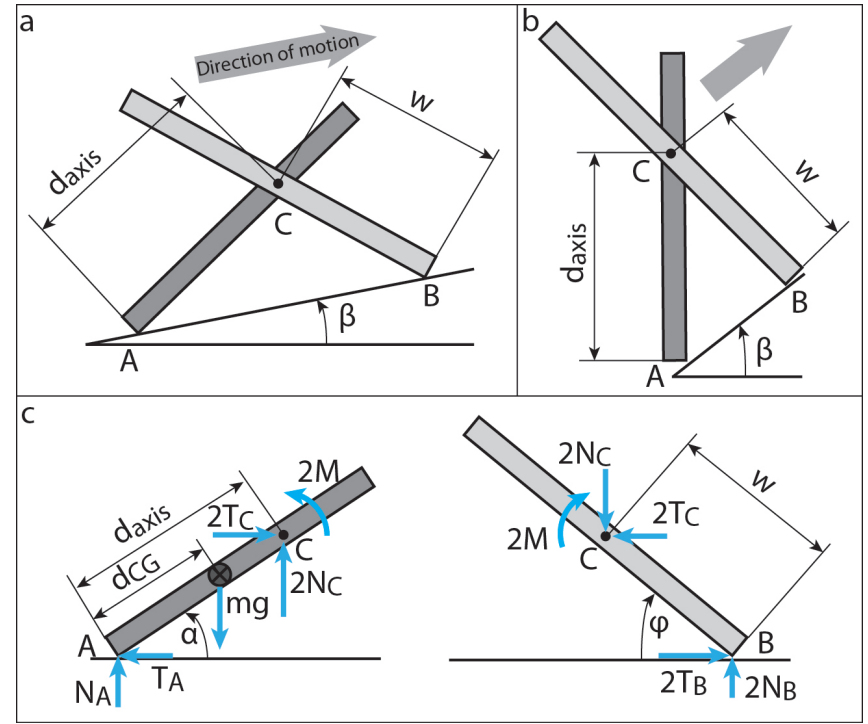

Fig. 6: (a) Schema of the robot seen from the side on a slope with dimension parameters; the center part in dark grey and the wing in light grey. (b) Limit of stability; for bigger values of $\beta$ or $w$, the robot would fall backwards. (c) Free body diagram; center part on the left, and wings on the right.

The second important point to consider is that the wings do not slip on the ground, the dynamic friction force $T=\mu N$ at point $\mathrm{A}$ should be smaller than at point $\mathrm{B}$, so that contact point A slips on the ground but not contact point B (see Fig. 6):

$$
\mu_{A} N_{A}<\mu_{B} N_{B}
$$

To evaluate this condition the model is separated into two bodies (see Fig. 6.c). We make the hypothesis that the mass of the wings can be neglected compared to the total mass of the robot. As there are two wings and two motors, the contact forces at point $\mathrm{B}$ and $\mathrm{C}$ are doubled. Ideally, the contact points of the wings (point B in Fig. 6) should not slip on the ground. From Newton's and Euler's equations applied on this system (at angle $\varphi=0^{\circ}$ ) we can deduce:

$$
\begin{aligned}
& N_{A}=m g\left(1-\frac{d_{C G}}{d_{\text {axis }}+w}\right) \\
& N_{B}=m g \frac{d_{C G}}{2\left(d_{\text {axis }}+w\right)}
\end{aligned}
$$

which lead to the following inequality:

$$
\frac{d_{a x i s}+w}{d_{C G}}<1+\frac{\mu_{B}}{2 \mu_{A}} .
$$

The friction between the ground and the robot has an influence on the admitted limit values of the parameters. The friction coefficient at the contact point on the center part A should be reduced to a minimum to permit a slipping-less rotation of the wings.

When the constraint (5) is satisfied, the transversal contact force is determined by the friction at point $A$ and can be used to evaluate the torque required by the motors:

$$
T_{A}=2 T_{B}=2 T_{C}=\mu_{A} N_{A} .
$$

Hence, the torque for one motor (at $\varphi=0^{\circ}$ ):

$$
M=\frac{1}{2} m g \frac{d_{C G}}{1+\frac{d_{a x i s}}{w}} .
$$

For higher values of $\varphi$, the required torque depends also on the friction coefficient between the contact point of the robot A and the ground. For a good efficiency of the locomotion, the required torque should be as small as possible and the covered distance per revolution should be as long as possible. The length of the covered distance $l$ per rotation of the wings is proportional to $w$, assuming that the wings do not slip: $l=4 w$. Supposing that the robot moves forwards with a certain mean speed $v$, the required average rotational speed of the wings $\Omega$ is:

$$
\Omega=v \frac{2 \pi}{l}=v \frac{\pi}{2 w} .
$$

\section{Morphology Optimization}

The theoretical analysis presented above gives guidelines for the design of the geometry of the platform, however many different combinations of the different parameters (taper ratio, swept angle, and inner ratio) satisfy the above conditions. Therefore, in order to optimize the morphology of the robot, it was modelled in a physics based simulator, using the Open Dynamic Engine (ODE) library, and the different parameters were optimized for maximizing the speed of the robot in the ground locomotion mode. 


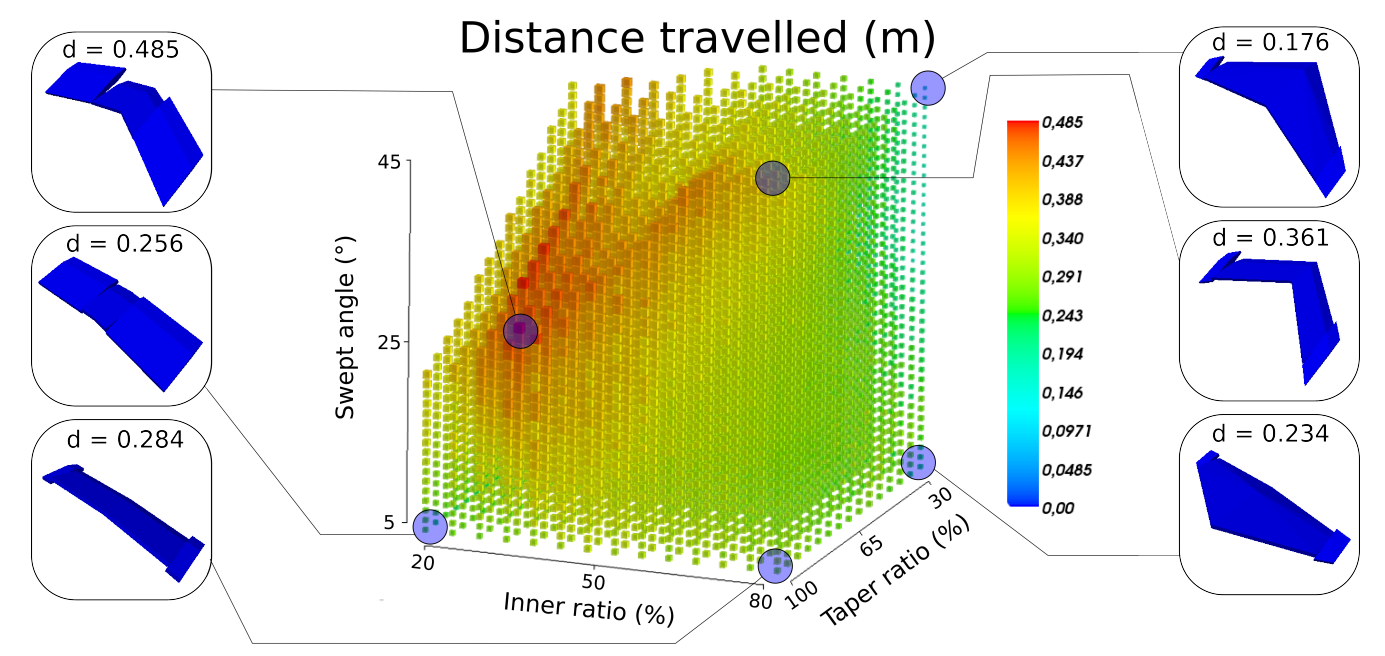

Fig. 7: Distance travelled for one revolution of the wings, and sample set of morphologies. The swept angle is the angle $\theta$ shown in Fig. 5, the Taper ratio is ratio between the tip chord and the root chord $T=C_{t} / C_{r}$, and the Inner ratio is the ratio between the distance $d$ (distance between the center of the robot and the rotating wing) and the half wing span $I=2 d / b$.

Figure 7 shows the distance travelled for the different configurations. Each point represents a different geometry of the robot and the color represents the travelled distance for one complete revolution of the wings. The $\mathrm{x}$ axis represents the inner ratio (from 20 to $80 \%$ ), the y axis the taper ratio (from 30 to $100 \%$ ), and the $\mathrm{z}$ axis the swept angle (from $5^{\circ}$ to $45^{\circ}$ ). Points that are missing on the graph are geometries where the axis of rotation of the wings would have been outside of center part of the robot, and thus are not evaluated. This figure also shows the extreme configurations of the robots. From the graph we can see that the best results are obtained for a medium swept angle (between $20^{\circ}$ and $\left.30^{\circ}\right)$, a large taper ratio $(\geqslant 65 \%)$, and a small inner ratio $(\leqslant 50 \%)$. The point with the highest value has the following parameters: inner ratio $35 \%$, taper ratio $95 \%$, and swept angle $26^{\circ}$. The prototype that we built to validate this concept was thus designed using these same parameters, and is presented in the next section. These parameters do not have a significant impact on the forward flight performance of the robot. However, the airfoil profile and the placement of the CG must be carefully adapted to the wing geometry.

\section{PROTOTYPING}

This section presents the important aspects of the mechanical design of the DALER, its ground and forward flight locomotion capabilities.

\section{A. Mechanical Design}

For the flight mode of locomotion the wings have to be sufficiently rigid in order to sustain the lift force and for the ground mode it must sustain high torsion forces during walking. The prototype was thus $3 \mathrm{D}$ printed in plastic, the wings structure was optimized for rigidity and weight. The total weight of the robot is $450 \mathrm{~g}$ for a wing span of $60 \mathrm{~cm}$.

The rotation of the wings must be locked during flight, thus a locking mechanism is used to freeze the rotation of the wings. Figure 8.C and D show this locking mechanism,
TABLE I: Friction coefficients.

\begin{tabular}{lcccc}
\hline & Parquet & Carpet & Road & Grass \\
\hline Robot center $\mu_{A}$ & 0.3 & 0.4 & 0.5 & 0.5 \\
Wings $\mu_{B}$ & 0.45 & 1.8 & 1.7 & 1.6 \\
$1+\frac{\mu_{B}}{2 \mu_{A}}$ & 1.75 & 3.25 & 2.7 & 2.6 \\
\hline
\end{tabular}

and Fig. 8.B shows the axis of rotation of the wing, the motor that is hidden inside the center of the robot, the gears, and the slip ring. The slip rings prevent the servo-motors' cables, used for the flaps on the wings, to be twisted.

Finally, Fig. 8.A and D show the small hooks that were added on the wings to increase the friction with the ground, they are covered with a very rough tape. Table I gives the friction coefficients on different surfaces for the wings and for the center of the robot. The relation between the parameters $\frac{d_{a x i s}+w}{d_{C G}}$ for this robot is equal to 2.5. Therefore, we can see that equation (5) is satisfied for all surfaces except for the parquet that is very slippery. The robot can still walk on the parquet but is less efficient than on the other surfaces, since it is slipping only on a small portion of each step.

\section{B. Ground and Air Locomotion Capabilities}

The flight and walking abilities of the DALER prototype can be seen in the accompanying video. This prototype is capable to walk on different terrains, such as on parquet, on carpet, in snow, on a road, and on grass (see Fig. 9). When the wings rotate synchronously at cruise speed the robot can go at $0.2 \mathrm{~m} / \mathrm{s}$ (or $0.66 \mathrm{BL} / \mathrm{s}$ ) and can rotate on spot at $25^{\circ} / \mathrm{s}$.

It can fly at about $14 \mathrm{~m} / \mathrm{s}$, is robust to landings at that speed, and the autonomy of such flying wing is about 30 minutes in forward flight. The hover flight with contrarotating propellers and flaps in the airflow was implemented ion the other prototype (see Fig. 3). This prototype can hover for about 10 minutes and is very stable and manoeuvrable. This solution will thus integrated in the next version of the 


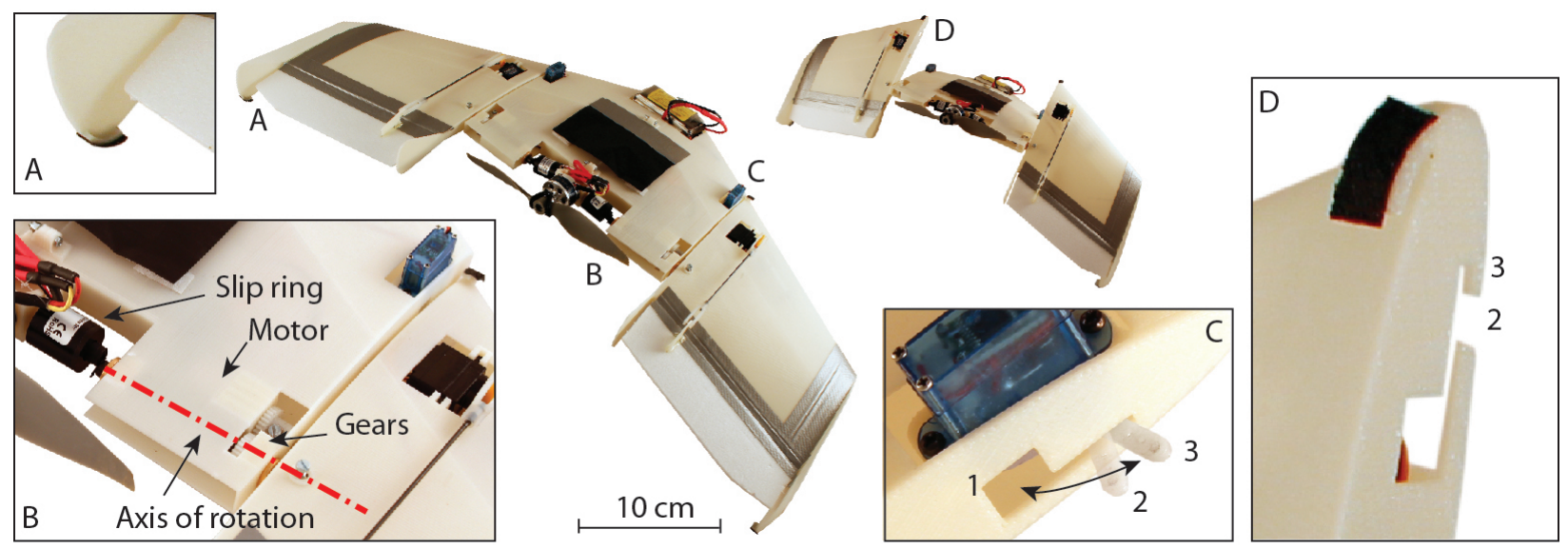

Fig. 8: (A) and (D) small hooks added on the wings and covered with rough tape to increase the friction with the ground. (C) locking mechanism; the servo-motor arm is in position 1 (arm retracted) when the robot walks on the ground, then it can be open to position 2 and the wings rotate until the arm of the servo-motor slices into the corresponding slot 2 (shown in D), and finally the wings are locked when the arm is in position 3. (B) Slip ring, motor, gears and axis of rotation.

DALER prototype. To transition between hover and forward flight the robot can either accelerate vertically to gain speed and slowly pith down, or it can perform a dive to gain speed and then pitch up (we achieved this with a third prototype).

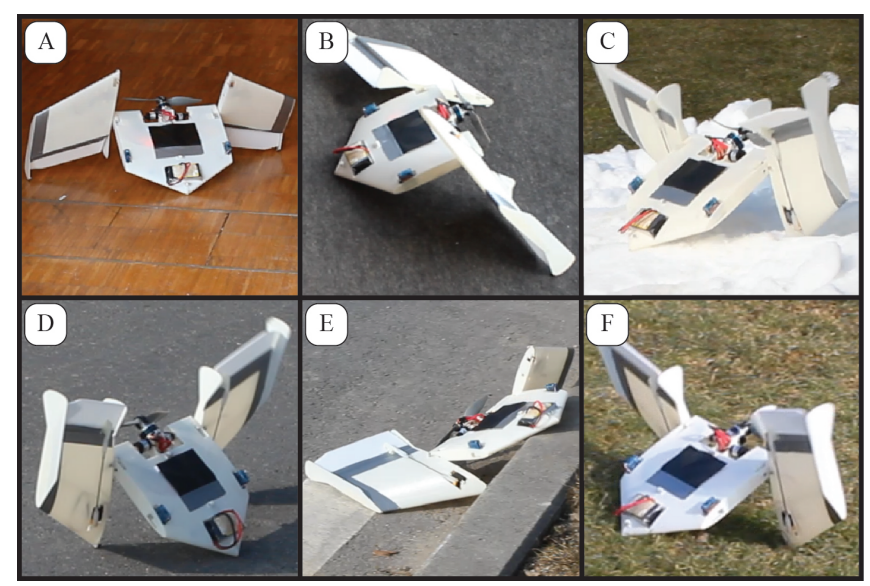

Fig. 9: Ground locomotion on different types of terrain. (A) On parquet, (B) on carpet, (C) in snow, (D) on a road, (E) going down a step, and $(\mathrm{F})$ on grass.

\section{CONCLUSION AND FUTURE WORK}

We designed a platform that is capable of multi-modal air and ground locomotion, and the use of the wings as whegs allows to keep the total mass of the platform low. Furthermore, the platform is effective in both modes of locomotion. Hover flight was experimented in an other prototype and future work will be done on enabling the DALER platform with hovering capabilities and adaptive deployable wings, in order to achieve the scenario presented in the introduction.

\section{ACKNOWLEDGMENTS}

This work was supported by the Swiss National Science Foundation through the National Centre of Competence in Research Robotics.

\section{REFERENCES}

[1] T. Chung, G. Hollinger, and V. Isler, "Search and pursuit-evasion in mobile robotics: A survey," Autonomous robots, vol. 31, no. 4, pp. 299-316, 2011.

[2] S. Tadokoro, Rescue Robotics: DDT Project on Robots and Systems for Urban Search and Rescue. Springer Verlag, 2009.

[3] H. Tennekes and H. Tennekes, The simple science of fight: from insects to jumbo jets. The MIT Press, 2009.

[4] D. Lentink and M. Dickinson, "Biofluiddynamic scaling of flapping, spinning and translating fins and wings," Journal of Experimental Biology, vol. 212, no. 16, p. 2691, 2009.

[5] A. Kossett, R. D'Sa, J. Purvey, and N. Papanikolopoulos, "Design of an improved land/air miniature robot," in Robotics and Automation (ICRA), 2010 IEEE International Conference on. IEEE, 2010, pp. 632-637.

[6] M. Kovac, J. Zufferey, and D. Floreano, "Towards a self-deploying and gliding robot," Flying insects and robots, 2009.

[7] K. Peterson and R. Fearing, "Experimental dynamics of wing assisted running for a bipedal ornithopter," in Intelligent Robots and Systems (IROS), 2011 IEEE/RSJ International Conference on. IEEE, 2011, pp. 5080-5086.

[8] R. Bachmann, F. Boria, R. Vaidyanathan, P. Ifju, and R. Quinn, "A biologically inspired micro-vehicle capable of aerial and terrestrial locomotion," Mechanism and Machine Theory, vol. 44, no. 3, pp. 513526, 2009.

[9] M. García-París and S. Deban, "A novel antipredator mechanism in salamanders: rolling escape in hydromantes platycephalus," Journal of herpetology, vol. 29, no. 1, pp. 149-151, 1995.

[10] G. Taylor, A. Carruthers, T. Hubel, and S. Walker, "Wing morphing in insects, birds and bats: Mechanism and function," Morphing Aerospace vehicles and structures, p. 13, 2012.

[11] R. Quinn, D. Kingsley, J. Offi, and R. Ritzmann, "It has got whegs,' in 2002 IEEE International Conference on Robotics and Automation (ICRA02) Video Proceedings, 2002.

[12] M. Itasse and J. Moschettai, "Equilibrium transition study for a hybrid mav," in International Micro Air Vehicle Conference and Flight Competition, 2011 IMAV, oct. 2011.

[13] C. Melhuish, J. Welsby, and P. Greenway, "Gradient ascent with a group of minimalist real robots: Implementing secondary swarming," in Proceedings of the IEEE International Conference on Systems, Man and Cybernetics, vol. 2, 2002, pp. 509-514.

[14] S. Leven, "Enabling large-scale collective systems in outdoor aerial robotics," Ph.D. dissertation, EPFL, 2011.

[15] A. Klaptocz, G. Boutinard Rouelle, A. Briod, J.-C. Zufferey, and D. Floreano, "An indoor flying platform with collision robustness and self-recovery," in IEEE/RSJ International Conference on Robotics and Automation, 2010, to appear.

[16] L. J. Clancy, Aerodynamics. Pitman, 1975. 